\title{
Angle-Resolved Plasmonic Properties of Single Gold Nanorod Dimers
}

\author{
Jian Wu $\cdot$ Xuxing Lu $\cdot$ Qiannan Zhu $\cdot$ Junwei Zhao $\cdot$ Qishun Shen $\cdot$ Li Zhan $\cdot$ Weihai Ni
}

Received: 8 July 2014/Revised: 25 August 2014/ Accepted: 4 September 2014/Published online: 26 September 2014

(C) The Author(s) 2014. This article is published with open access at Springerlink.com

\begin{abstract}
Through wet-chemical assembly methods, gold nanorods were placed close to each other and formed a dimer with a gap distance $\sim 1 \mathrm{~nm}$, and hence degenerated plasmonic dipole modes of individual nanorods coupled together to produce hybridized bonding and antibonding resonance modes. Previous studies using a condenser for illumination result in averaged signals over all excitation angles. By exciting an individual dimer obliquely at different angles, we demonstrate that these two new resonance modes are highly tunable and sensitive to the angle between the excitation polarization and the dimer orientation, which follows $\cos ^{2} \varphi$ dependence. Moreover, for dimer structures with various structure angles, the resonance wavelengths as well as the refractive index sensitivities were found independent of the structure angle. Calculated angle-resolved plasmonic properties are in good agreement with the measurements. The assembled nanostructures investigated here are important for fundamental researches as well as potential applications when they are used as building blocks in plasmon-based optical and optoelectronic devices.
\end{abstract}

Keywords Plasmonics · Gold nanorods · Self-assembly

\section{Introduction}

In recent years, noble metal nanoparticles and their assembled nanostructures have attracted a great amount of

Electronic supplementary material The online version of this article (doi:10.1007/s40820-014-0011-7) contains supplementary material, which is available to authorized users.

\section{J. Wu $\cdot$ Q. Shen $\cdot$ L. Zhan $(\bowtie)$}

Department of Physics and Astronomy, Key Laboratory for Laser Plasmas (Ministry of Education), State Key Lab of

Advanced Optical Communication Systems and Networks, Shanghai Jiao Tong University, Shanghai 200240,

People's Republic of China

e-mail: lizhan@sjtu.edu.cn

J. Wu $\cdot$ X. Lu $\cdot$ Q. Zhu $\cdot$ J. Zhao $\cdot$ W. Ni ( $₫)$ Division of i-Lab, Key Laboratory for Nano-Bio Interface Research \& Collaborative Innovation Center of Suzhou Nano Science and Technology, Suzhou Institute of Nano-Tech \& Nano-Bionics, Chinese Academy of Sciences, Suzhou 215123, Jiangsu, People's Republic of China e-mail:whni2012@sinano.ac.cn attention because they show prominent and versatile optical properties in the visible range due to localized surface plasmon resonance (LSPR). In the assembled nanostructures, metal nanoparticles are placed close to each other, and the localized surface plasmon of individual nanoparticles can be coupled together giving rise to so-called "hot spots", which are important for fundamental researches as well as potential applications in optics [1-4], chemistry [5-7], or electronics [8].

To date, studies based on calculations [8-11] and experiments [12-16] have been performed on the plasmonic coupling in metal nanoparticle-assembled dimer structures. Created by wet-chemical assembly methods, the dimer structures have much smaller gap distance than what can typically be achieved using electron-beam lithography fabrication methods ( $>5 \mathrm{~nm}$ ) [17]. For a typical nanorod dimer structure with small gap distance, degenerate plasmonic dipole modes of individual nanorods will couple together to produce two new plasmonic resonances, antibonding and bonding modes respectively at higher and lower energies, whose separation is approximated by the 
Simpson-Peterson equation [9]. The symmetry breaking in the dimer structures induces the appearance of the normally dark antibonding modes as well as the dark-to-bright mode conversion $[18,19]$. The two new resonance modes are highly tunable and sensitive to polarization orientation, which enables individual dimers to act as efficient orientation sensors [1, 20, 21]. However, these studies on individual nanorods are usually performed using a condenser for illumination with high numerical apertures where the signal obtained is averaged over all excitation angles. Little effort was made to study the effects of the polarization of excitation with respect to the dimer orientation, and therefore contribution from individual resonance modes cannot be discriminated.

In this paper, we performed a systematic study on the angle-resolved plasmonic properties of single gold nanorod dimer structures by exciting the dimer obliquely at specific or various angles. Gold nanorod dimer structures are created using cysteine (CYS) as linking molecules in aqueous solutions. V-shaped dimer structures are formed by depositing the assemblies from solutions onto a substrate. Strong plasmonic coupling occurs in these dimer structures, which results in the hybridized bonding and antibonding resonance modes. Single-particle dark-field scattering experiments indicate that the modes both are highly sensitive to the angle $\varphi$ between the excitation polarization and the orientation of the dimer. The scattering intensity follows a typical $\cos ^{2} \varphi$ dependence due to the decomposition of the excitation electric field vector along either the bisector of the dimer for the radiative antibonding mode or its orthogonal direction for the radiative bonding mode. Effect of the angle between the two nanorods in the dimer on the scattering is also investigated by combining scanning electron microscope (SEM) and single-particle scattering spectroscopy. Both analytical and numerical methods are used in the calculations. The resonance wavelengths as well as the refractive index sensitivities are found independent of the structure angle. The calculated results are in good agreement with the measurements. Knowledge gained is helpful for the design of more complicated plasmonic structures, such as Fano and chiral structures. It is of particular interest for applications because these dimer structures can be used as building blocks in plasmon-based optical and optoelectronic devices.

\section{Results and Discussion}

Gold nanorods were prepared using the seed-mediated growth method, and they are stabilized with cetyltrimethylammonium bromide (CTAB) in aqueous solutions. Figure 1a shows a transmission electron microscope (TEM) image of the as-synthesized gold nanorods. The average diameter, length, and aspect ratio of the nanorods are $23.6 \pm 1.8 \mathrm{~nm}, 69.3 \pm 4.9 \mathrm{~nm}$, and $2.9 \pm 0.3 \mathrm{~nm}$, respectively. The gold nanorods show a longitudinal surface plasmon resonance at $717 \mathrm{~nm}$ in aqueous solutions (Fig. S1). Some of the gold nanorods show a slightly dog-bone shape. Compared to gold nanorods with ideal shape, the dog-bone nanorods possess a slightly red-shifted resonance wavelength [22]. The assembly of nanorods was performed by adding CYS to the solution as linking molecules, which is recorded spectroscopically (Fig. S1). Figures $1 b-d$ show TEM images of representative dimer structures where two nanorods are connected at their ends through the linking molecules. The gap distance between the two nanorods was estimated to be about $1 \mathrm{~nm}$ according to the high-resolution TEM image (Fig. 1e). The gap distance between the two adjacent nanorods is largely determined by the linking molecule (CYS) and influenced by the CTAB surfactant bilayer. Based on the 3D construction of CYS molecule, the length starting from the thiol group and ending with the carboxyl group is approximately $0.6 \mathrm{~nm}$. Because the molecular link involves two CYS molecules in an end-toend fashion [23], gap distance is estimated to be $1.2 \mathrm{~nm}$. This value is consistent with both TEM measurements and literature reports $[4,24,25]$. The assembled nanorods were then deposited from the solution onto a glass substrate. Figure 1f shows a SEM image of the nanorod dimers on the substrate. As shown in the inset, a dimer structure (highlighted in the blue circle) was identified with an angle $\theta=80^{\circ}$ between the long axes of the two nanorods. The length and diameter of the two nanorods were measured to be $l_{1}=71 \mathrm{~nm}$ and $d_{1}=24 \mathrm{~nm}$ (on the left), and $l_{\mathrm{r}}=68 \mathrm{~nm}$ and $d_{\mathrm{r}}=23 \mathrm{~nm}$ (on the right). The gap distance can hardly be measured under SEM because excess electron dose may damage the structure when SEM is working under highmagnification modes.

The dark-field scattering image and spectra of the individual gold nanorod dimers were measured using an Olympus BX53 optical microscope integrated with an Acton SpectraPro SP2750 monochromator and a Princeton Instruments PyLon 400BR charge-coupled device (CCD), which was cooled by liquid nitrogen to $\sim 120^{\circ} \mathrm{C}$. The dimer structures deposited on glass slides were illuminated by white light from a $100 \mathrm{~W}$ Halogen lamp. The scattered light was collected with a long working distance 50X objective (N.A. $=0.5)$ and reflected to the entrance slit of the monochromator for imaging and spectroscopic measurements. Scattering spectra from individual dimers were calibrated by subtracting background spectra taken from the adjacent regions containing no nanostructures [26]. Single-particle measurements were performed by correlating the SEM and optical images. At the same area of the SEM image (Fig. 1f), a dark-field scattering image was 

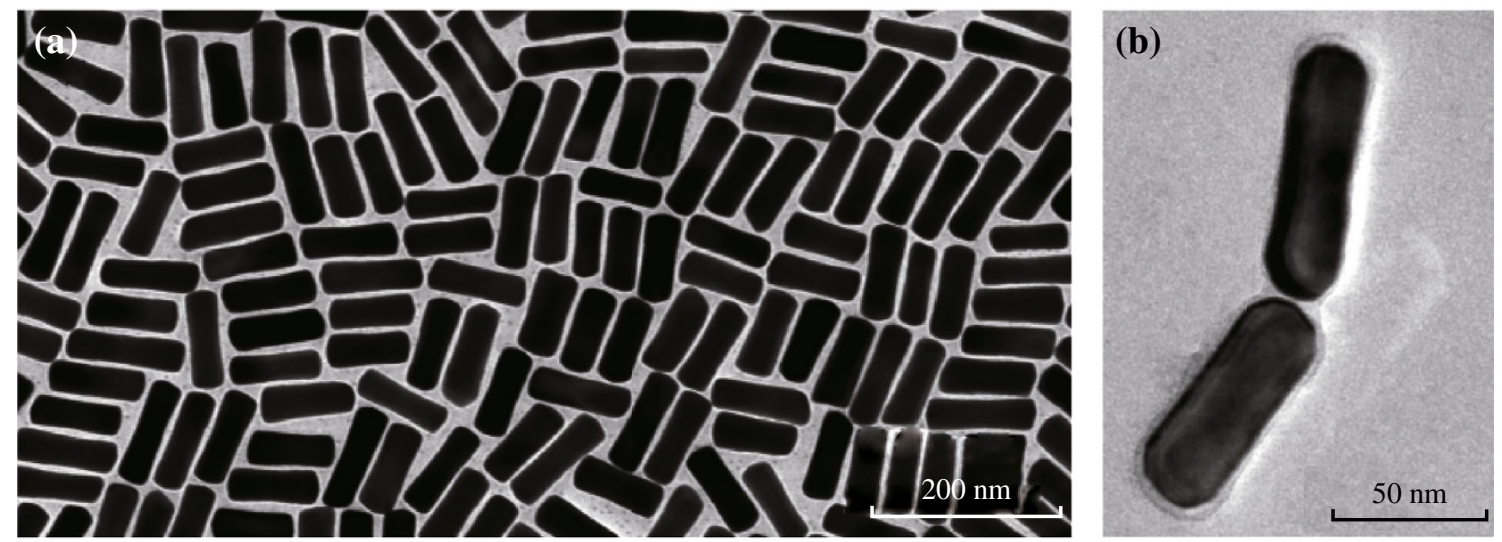

(c)

(d)
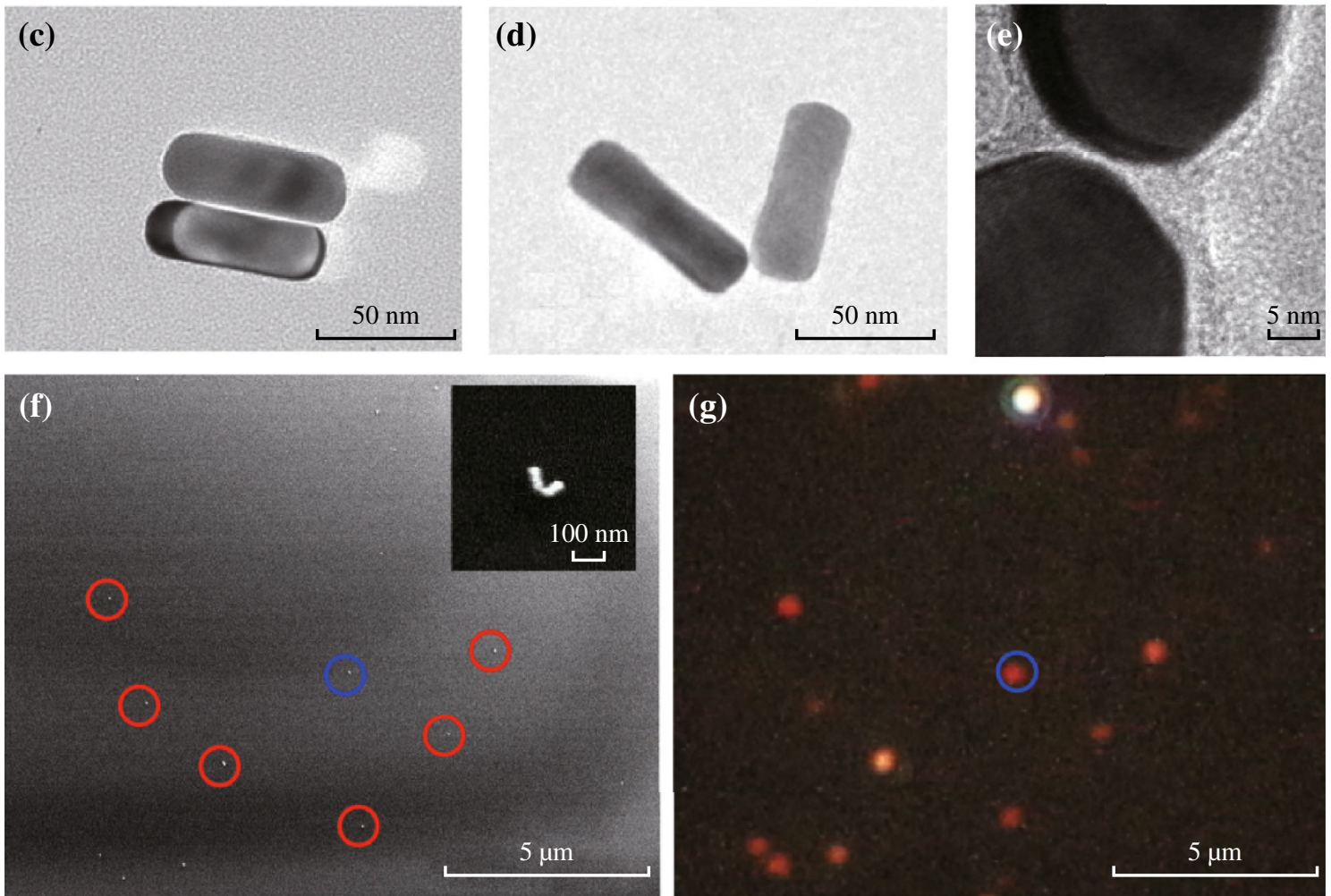

Fig. 1 SEM and optical characterizations of gold nanorod assemblies. a TEM image of gold naonrods $(69.3 \pm 4.9 \times 23.6 \pm 1.8 \mathrm{~nm}$, with an aspect ratio of $2.9 \pm 0.3)$. b-d Representative TEM images of three assembled gold nanorod dimers. The angles between the two nanorods are $0^{\circ}, 70^{\circ}$, and $149^{\circ}$, respectively. e High-resolution TEM image for the gap of the gold nanorod dimer in (b), indicating the gap distance between the two nanorods as about $1 \mathrm{~nm}$. f SEM image for the gold nanorod dimers deposited on a cover glass substrate. The inset shows a SEM image for the dimer highlighted in the blue circle at high magnification. $\mathbf{g}$ Dark-field scattering image of the same area shown in SEM image (f). (Color figure online)

taken for the gold nanostructures and shown in Fig. 1g. The diffraction-limited bright spots represent individual gold nanostructures and can be directly correlated with the nanostructures identified on the SEM image.

Figure 2a shows the schematic design of the experimental setup for the angle-resolved measurements. $\theta$ is defined as the angle between the long axes of the two nanorods in the dimer. The dimer is excited by an incident light at an incident angle $\psi$. The substrate can be rotated clockwise around the z-axis by an angle $\varphi$. Incident light with P- (blue double-headed arrow) and S-polarizations (red double-headed arrow) is indicated as the excitation source for the scattering measurements. Under the excitation of the incident light with P- and S-polarization, the scattering spectra of a dimer with $\theta=80^{\circ}$ was measured at $\psi=60^{\circ}$. By continuously rotating the substrate starting from $\varphi=0^{\circ}$ to $180^{\circ}$ at a step of $10^{\circ}$, scattering spectra from the same dimer structure were respectively recorded under the excitation of S- and P-polarizations, which is partially presented in Fig. $2 b$ from bottom to top. Two 

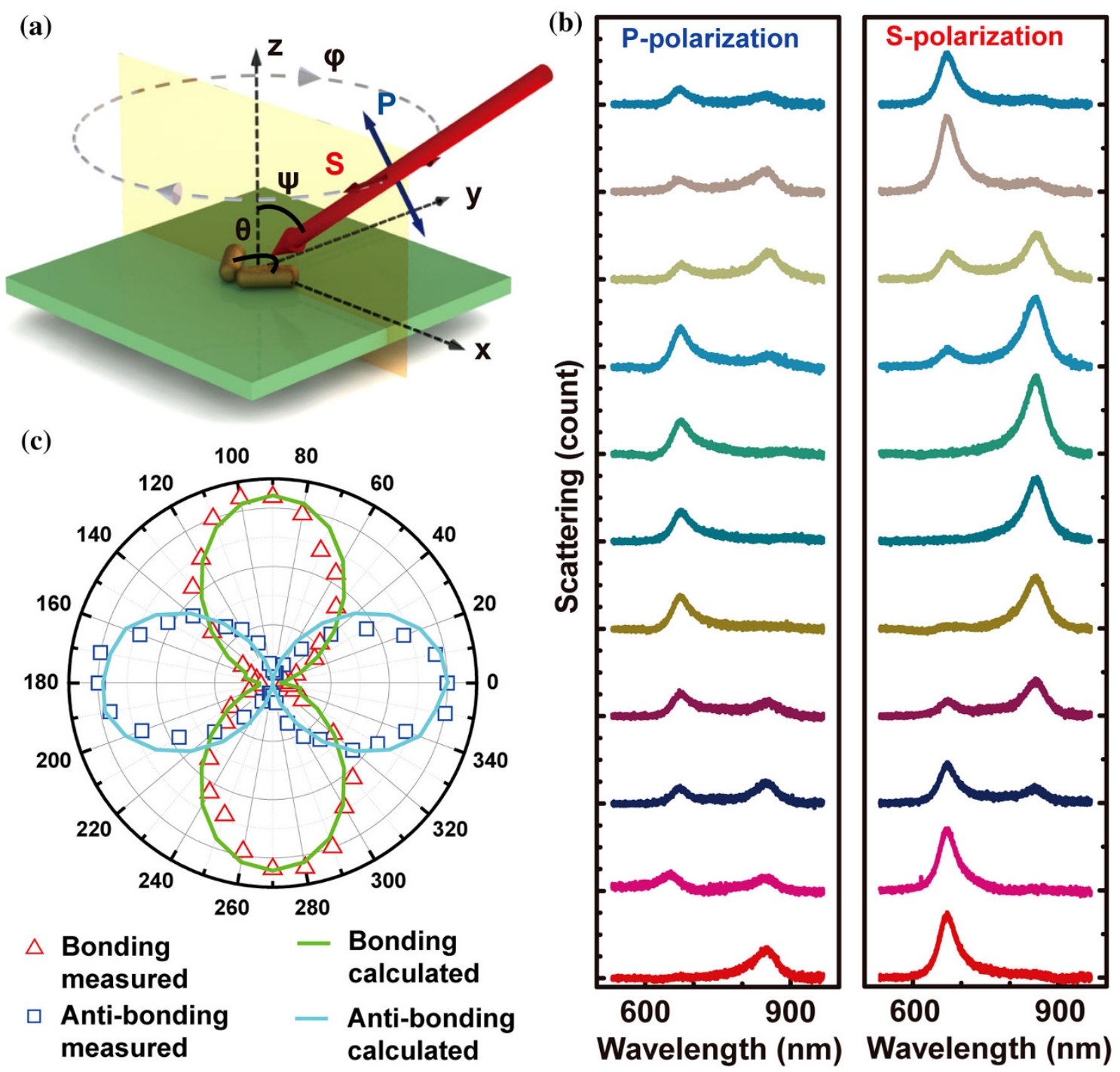

Fig. 2 Angle-resolved plasmonic properties of an individual dimer. a Schematic design of the experimental setup for the angle-resolved measurements. A dimer with structure angle of $80^{\circ}$ is investigated. The dimer is excited obliquely by an incident light at an incident angle $\psi$. The substrate can be rotated clockwise around the z-axis by an angle $\varphi$. Incident light with P- (blue double-headed arrow) and S-polarizations (red double-headed arrow) is indicated as the excitation source for the scattering measurements. b Scattering spectra from the dimer structure (inset, Fig. 1f) recorded respectively under the excitation of S- and P-polarizations. From bottom to top: scattering spectra with $\varphi=0^{\circ}, 20^{\circ}, 40^{\circ}, 60^{\circ}$, $80^{\circ}, 90^{\circ}, 100^{\circ}, 120^{\circ}, 140^{\circ}, 160^{\circ}$, and $180^{\circ}$. c S-polarization peak intensity of the two resonance peaks plotted as a function of $\varphi$. Measured peak intensities (squares and triangles are for peak intensities at 672 and $853 \mathrm{~nm}$, respectively.) are compared with calculation. (Color figure online)

peaks can be clearly identified at $672 \mathrm{~nm}$ and $853 \mathrm{~nm}$ in the spectra. With the increase of $\varphi$, as we can see for the S-polarization, the peak at $672 \mathrm{~nm}$ reaches a maximum when the polarization of excitation is parallel to the bisector of the nanorod dimer $\left(\varphi=0^{\circ}\right)$, and decreases to zero when it is perpendicular to the bisector $\left(\varphi=90^{\circ}\right)$. The peak at $853 \mathrm{~nm}$ just shows an opposite behavior. This periodic evolution can be clearly observed in Fig. 2c, where the peak intensity of the two resonance peaks is plotted as a function of $\varphi$. The behaviors of the two resonances show $90^{\circ}$ difference in the angle dependence, suggesting that the orientations of these two resonance modes are orthogonal to each other.
In order to understand the observed angle dependence of the plasmonic properties, both numerical and analytical methods are used for the theoretical simulations. For a typical scattering spectrum obtained with specific excitation polarization (Fig. 3a), the scattering peaks can be assigned to plasmonic resonance modes through calculating charge distribution profiles at the resonance wavelengths. Figure $3 \mathrm{~b}$ shows the charge distribution profiles obtained using finite difference time domain (FDTD) method [27, 28]. Under the excitation of light with S-polarization, the individual dipoles oscillate symmetrically at $672 \mathrm{~nm}$ and produce a resultant dipoles along the bisector of the dimer (Fig. 3b upper-left), which give rise 

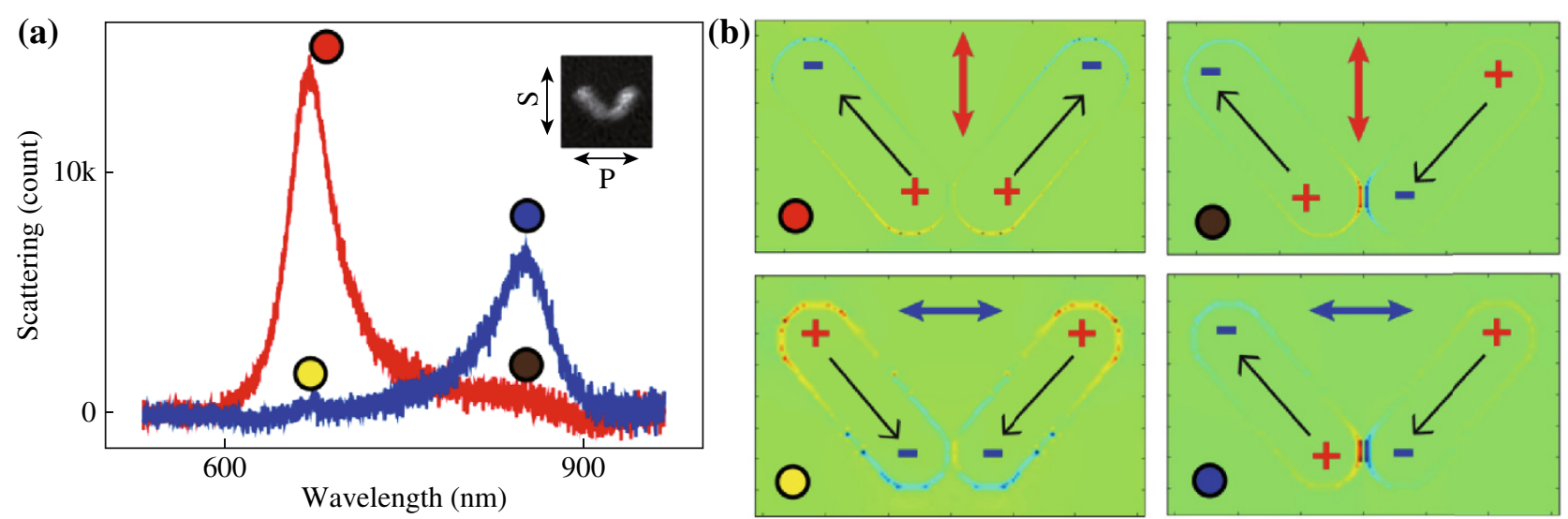

(c) Anti-
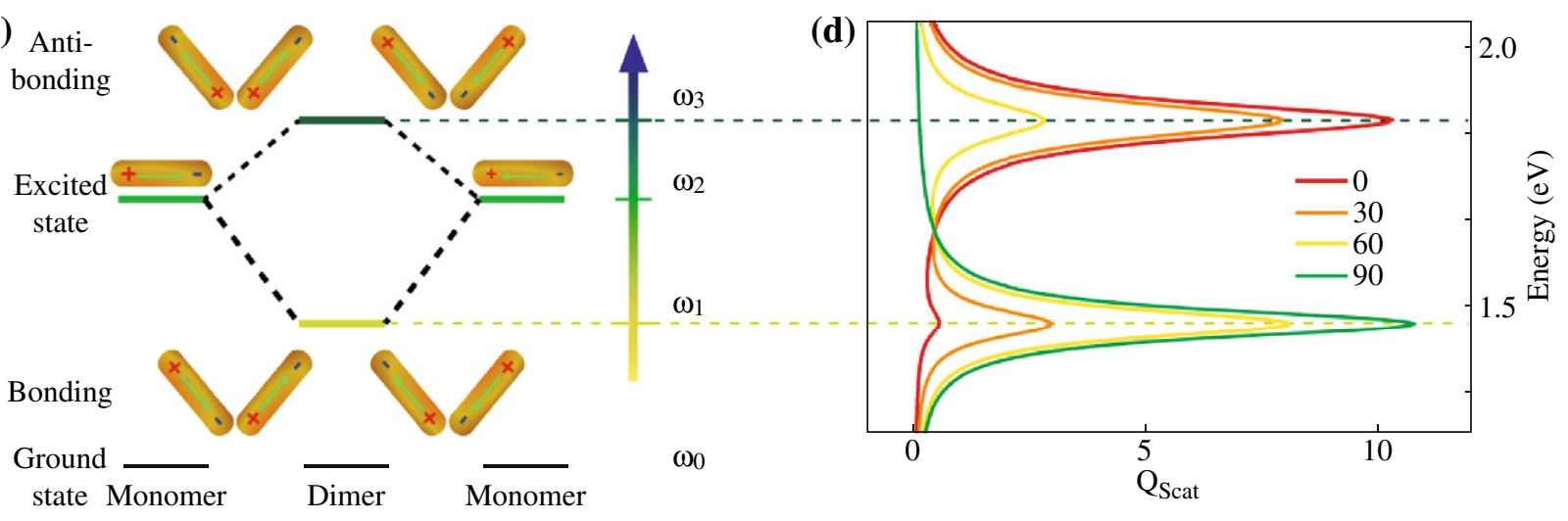

Fig. 3 Plasmonic hybridization of the gold nanorod dimer. a A typical scattering spectrum obtained with specific excitation polarization. b Charge distribution profiles obtained using FDTD method. The colors indicate the relative sign of the charges. Upper-left S-polarization at $672 \mathrm{~nm}$. Upper-right S-polarization at $853 \mathrm{~nm}$. Lower-left P-polarization at $672 \mathrm{~nm}$. Lower-right P-polarization at $853 \mathrm{~nm}$. c Plasmonic hybridization theory scheme depicting the analogous hybridized excited energy. d Calculated scattering coefficient is plotted as a function of energy at various $\varphi=0^{\circ}, 30^{\circ}, 60^{\circ}$, and $90^{\circ}$. (Color figure online)

to the corresponding resonance scattering peak in the spectrum (red circle). On the contrary, at $853 \mathrm{~nm}$, they oscillate anti-symmetrically and tend to cancel each other (Fig. 3b upper-right), which give rise to a zero resultant dipole and dark in scattering (brawn circle). As for the $P$ polarization, symmetric and anti-symmetric oscillations of the individual dipoles (Fig. 3b lower-left and lower-right) result in dark (yellow circle) and bright in scattering (blue circle), respectively. To get deeper insights into the physics behind plasmonic properties of the gold nanorod dimer, we conceive a simple plasmonic hybridization picture for the description of the electromagnetic behavior of the gold nanorod dimer. As shown in Fig. 3c, when the two gold nanorods are placed close to each other, due to strong plasmonic coupling the degenerate plasmonic resonance of individual gold nanorods is split into two hybridized resonance modes at lower and higher energies, corresponding to bonding and antibonding plasmonic modes, as well as anti-symmetric and symmetric collective charge density oscillations, respectively $[15,29]$. The excitation efficiencies of these two hybridized modes are highly dependent on the relative orientation between the dimer structure and the polarization of the excitation light. At an arbitrary angle $\varphi$, the excitation light with specific polarization can be decomposed into two parts. One is along the bisector of the nanorod dimer which is related to the excitation of the radiative antibonding mode and the other is orthogonal and related to the radiative bonding mode. The fraction of the decomposition actually determines the relative height of the scattering at the two resonance energies.

Coupled dipole approximation (CDA) method was employed to investigate the angle dependence of the plasmonic properties of the gold nanorod dimer. Each gold nanorod is approximated as a prolate ellipsoid with a polarization tensor $\vec{\alpha}_{j}=T_{j}^{-1} \overleftrightarrow{\alpha}_{0} T_{j}(j=1,2)$, where $\overleftrightarrow{\alpha}_{0}$ is diagonal matrix with one longitudinal element $\alpha_{\mathrm{L}}$ and two transverse elements $\alpha_{\mathrm{T}}$ in the principal axes system of the rods, and $T_{j}$ is the rotation matrix which relates the ellipsoid frame with the lab frame. By using Rayleigh-Gans approximation, the polarization elements can be given as $[30,31]$ : 
$\alpha_{j}=\frac{V}{4 \pi} \frac{\varepsilon-\varepsilon_{m}}{\varepsilon_{m}+\left(\varepsilon-\varepsilon_{m}\right) L_{j}}$,

where $V$ is the volume of the nanoparticle, $\varepsilon_{m}$ is the dielectric constant of the surrounding medium, and $\varepsilon$ is the dielectric function of gold [32].

The depolarization factors $L_{j}$ are defined as

$$
\begin{aligned}
L_{L} & =\frac{1-e^{2}}{e^{2}}\left[\frac{1}{2 e} \ln \left(\frac{1+e}{1-e}\right)-1\right] \\
L_{T} & =\frac{1-L_{L}}{2}
\end{aligned}
$$

where the eccentricity $e$ is defined as $e=\sqrt{1-b^{2} / a^{2}}$, and $a$ and $b$ are the major and minor radii of the ellipsoid, respectively $[30,31]$. The polarization of each ellipsoid can be expressed as [33]:

$\mathbf{p}_{j}=\overleftrightarrow{\alpha}_{j} \cdot\left(\mathbf{E}_{\mathrm{inc}, j}+\overleftrightarrow{\mathbf{G}}_{j, i} \cdot \mathbf{p}_{i}\right)$

where $\mathbf{E}_{\mathrm{inc}, i}=E_{0} \mathbf{e}_{(s, p)} \exp \left(i \mathbf{k} \cdot \mathbf{r}_{i}-i \omega t\right)$ is the electric field at $r_{i}$ due to the incident plane wave. The subscript label $\mathrm{S}$ and $\mathrm{P}$ denote two orthogonal polarized lights with $\mathrm{s}-$ and $\mathrm{p}$ polarization, respectively. The unit vectors of the s-polarization $e_{s}$ (perpendicular to the incident plane), p-polarization $e_{p}$ (parallel to the incident plane), and the wave vector $e_{k}$ were chosen to obey right-handed rule $\mathbf{e}_{k}=\mathbf{e}_{s} \times \mathbf{e}_{p} \cdot \overleftrightarrow{\mathbf{G}}_{j, i} \cdot \mathbf{p}_{i}$ is the electric field due to the dipole at position $\mathrm{r}_{\mathrm{i}}(i \neq j)$ [33]:

$$
\begin{aligned}
\overleftrightarrow{\mathbf{G}}_{j, i} \cdot \mathbf{p}_{i}= & \frac{\exp \left(i k r_{j i}\right)}{r_{j i}^{3}}\left\{\frac{\left(1-i k r_{j i}\right)}{r_{j i}^{2}} \times\left[3 \mathbf{r}_{j i}\left(\mathbf{r}_{j i} \cdot \mathbf{p}_{i}\right)-r_{j i}^{2} \mathbf{p}_{i}\right]\right. \\
& \left.-k^{2} \mathbf{r}_{j i} \times\left(\mathbf{r}_{j i} \times \mathbf{p}_{i}\right)\right\}
\end{aligned}
$$

where $\mathbf{r}_{j i}=\mathbf{r}_{j}-\mathbf{r}_{i}, r_{j i}=\left|\mathbf{r}_{j}-\mathbf{r}_{i}\right|$

Therefore, coupled equation for the dipole $\mathrm{P}_{\mathrm{j}}(j=1,2)$ can be written as

$\sum_{i=1}^{2}\left(\delta_{j, i} \overleftrightarrow{\mathbf{I}}-\overleftrightarrow{\alpha} \cdot \overleftrightarrow{\mathbf{G}}_{j, i}\right) \cdot \mathbf{p}_{i}=\overleftrightarrow{\alpha} \cdot \mathbf{E}_{\mathrm{inc}, j}$

By solving these equations for the unknown polarizations $P_{j}$, the extinction and absorption cross section can be calculated as:

$C_{\text {ext }}=\frac{4 \pi k}{\left|\mathrm{E}_{\mathrm{inc}}\right|^{2}} \sum_{j=1}^{2} \operatorname{Im}\left(\mathrm{E}_{\mathrm{inc}, j}^{*} \cdot \mathbf{p}_{j}\right)$

$C_{a b s}=\frac{4 \pi k}{\left|\mathbf{E}_{\mathrm{inc}}\right|^{2}} \sum_{j=1}^{2}\left\{\operatorname{Im}\left[\mathbf{p}_{j} \cdot\left(\alpha_{j}^{-1}\right)^{*} \cdot \mathbf{p}_{j}^{*}\right]-\frac{2}{3} k^{3} \mathbf{p}_{j} \cdot \mathbf{p}_{j}^{*}\right\}$

with the scattering cross-section $C_{s c a}=C_{e x t}-C_{a b s}$.

The scattering coefficient is calculated using the CDA method and plotted as a function of energy at various $\varphi$ in
Fig. 3d. As shown in the figure, the relative height of the scattering peaks at two resonance modes continuously change when $\varphi$ is increased. When the calculated angle dependence of the scattering intensity is compared with the measurement, a satisfactory agreement is found (Fig. 2c). Note that the intensity follows a typical $\cos ^{2} \varphi$ dependence $[4,15,34]$. This is because the excitation $E$-field vector can be decomposed along either the bisector of the dimer for the radiative antibonding mode or its orthogonal direction for the radiative bonding mode. Through straight forward triangular calculation, one can find the excitation of antibonding mode have a factor of $\cos \varphi$. In terms of intensity, the square of the $E$-field, the dependence of scattering intensity should then take a factor of $\cos ^{2} \varphi$.

We next investigate the plasmonic properties that are dependent on the structure angle $\theta$, the angle between the long axes of the two individual nanorods in the dimer. Figure 4 shows the scattering spectra from nanorod dimer structures with various $\theta$. By varying $\theta$ in the range from $0^{\circ}$ to $180^{\circ}$, the dimer structures exhibit antibonding and bonding resonance scattering peaks at about 650 and $850 \mathrm{~nm}$, respectively. This result is consistent with the previous findings that the two resonance peaks are almost fixed at certain wavelengths [4, 18, 19]. As for the peak intensity, the two polarization states show opposite dependence. In the case of S-polarization, the intensity of the antibonding resonance scattering peak decreases with the increase of $\theta$, and diminishes when $\theta=180^{\circ}$. On the contrary, that of the bonding resonance peak increases with the increase of $\theta$. In order to explain these experimental observations, SCUFF-EM, an open-source software implementation of the boundary-element method, was employed in the calculation. The gold nanorod was modeled as a cylinder with two hemispherical tips at both ends. The diameter and length of the gold nanorod are 24 and $69 \mathrm{~nm}$ respectively, and the gap was set at $1 \mathrm{~nm}$. Linearly polarized waves with the polarizations parallel $(\mathrm{S})$ or perpendicular $(\mathrm{P})$ to the symmetric plane of the dimer was used as excitation source in the calculations. The dielectric constant of bulk gold obtained from literature values was used [32]. Figures 5a and b show the calculated scattering cross sections for the dimer structures with various $\theta$ under the excitation of light with $\mathrm{S}$ - and P-polarizations, respectively. With the increase of the structure angle $\theta$, the scattering intensity of the antibonding mode gradually decreases while that of the bonding mode gradually increases, which is consistent with the experimental observations. The peak wavelengths of the two resonance modes, however, were almost unchanged at fixed values (Fig. 5c). This is because the hybridized energy is mainly dependent on the coupling strength that is determined by the gap distance [35]. Considering the dimer structures with varying structure angles possess the same gap 

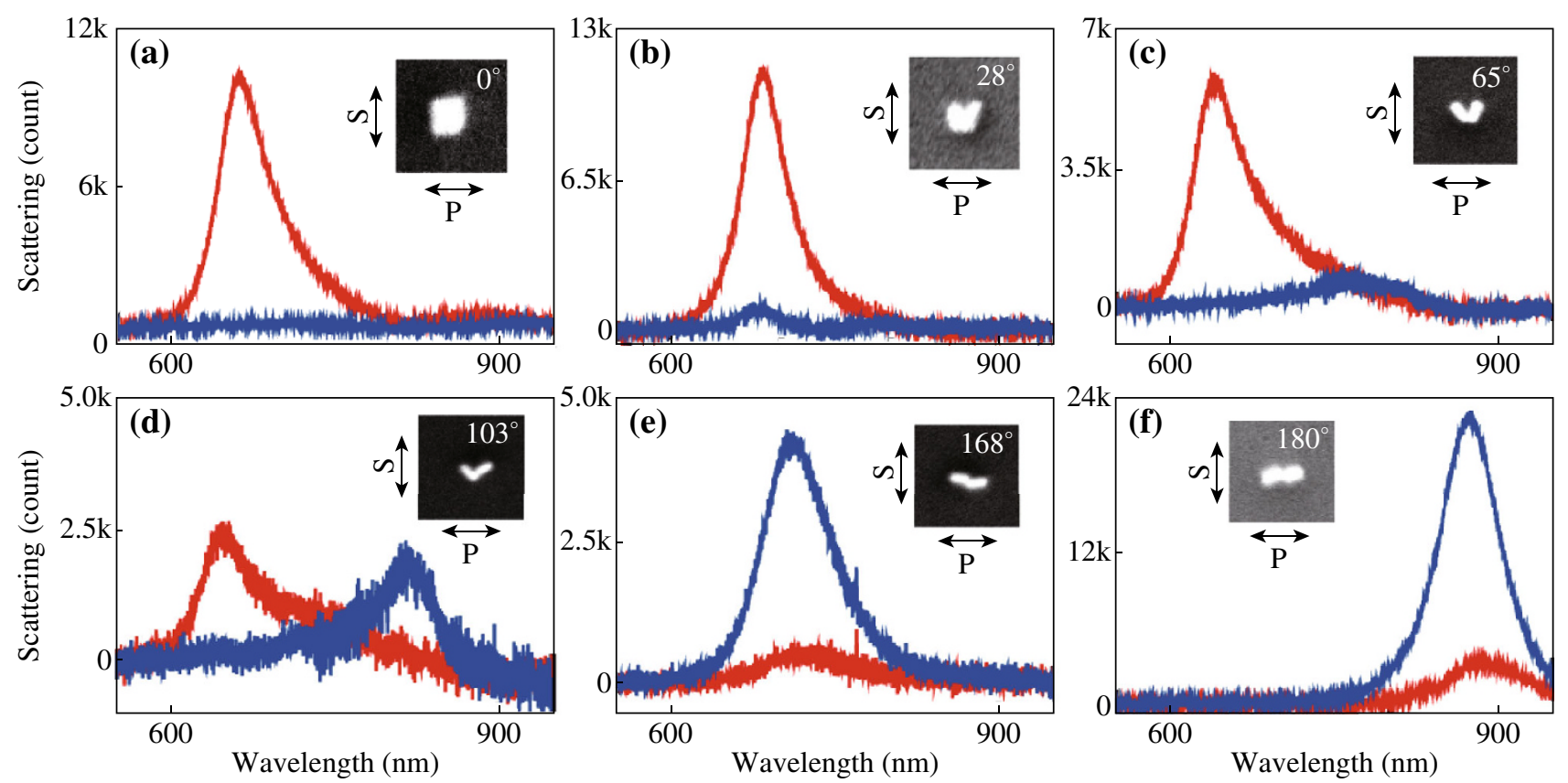

Fig. 4 Scattering spectra from nanorod dimer structures with various $\theta$. From a to f, the structure angles are $0^{\circ}, 28^{\circ}, 65^{\circ}, 103^{\circ}, 168^{\circ}$, and $180^{\circ}$, respectively. Zoomed-in SEM images of the dimer structures are shown in the inset. S- (red) and P-polarization (blue) are respectively parallel and perpendicular to the bisector of the nanorods. (Color figure online)
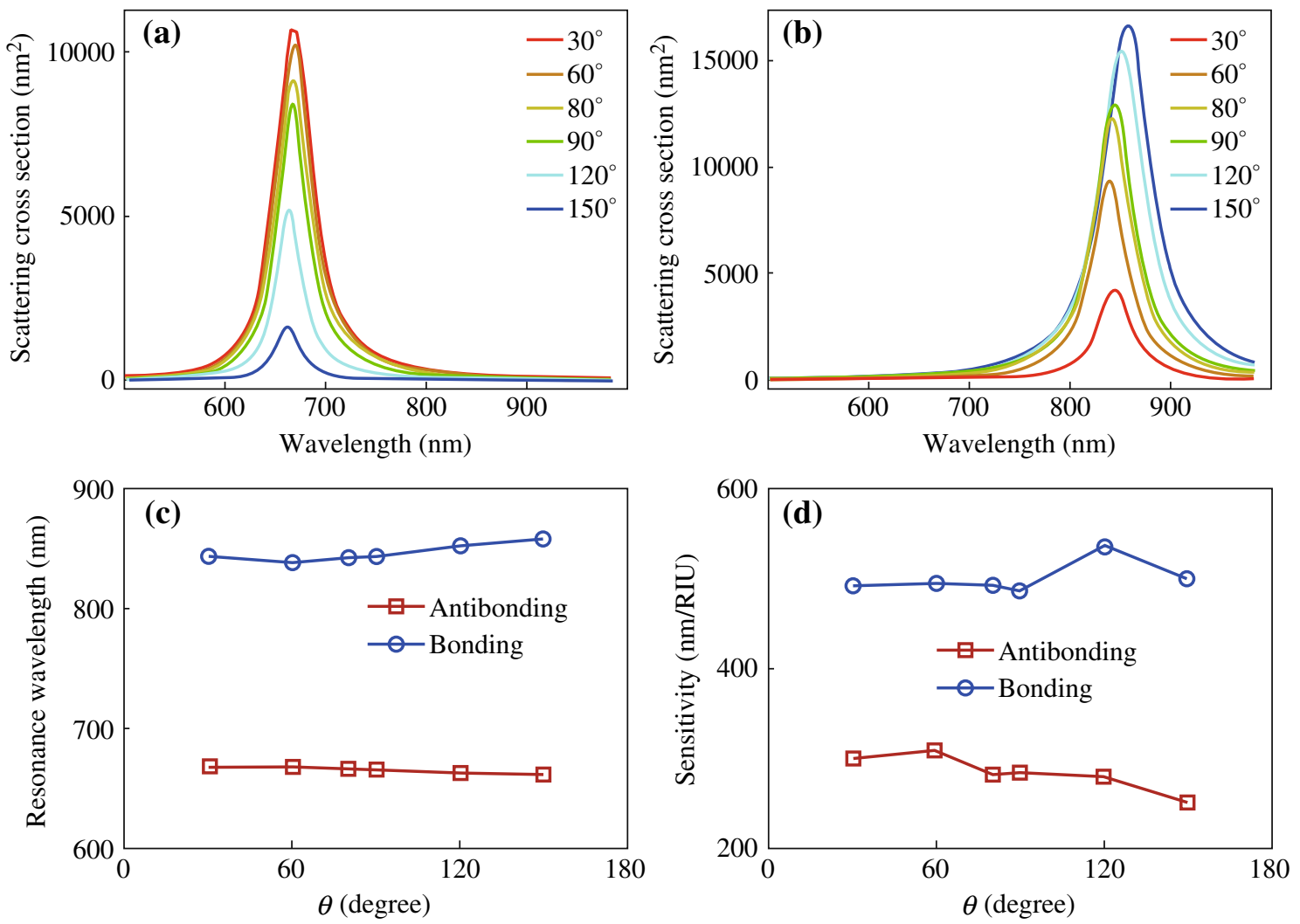

Fig. 5 Plasmonic properties of the dimer structures with various structure angle $\theta$. a Calculated scattering cross sections of the dimer under the excitation of light with S-polarization. b That for P-polarization. $\mathbf{c}$ Peak wavelengths of the two resonance modes as a function of structure angle. d Refractive index sensitivity as a function of structure angle 
distance, it is reasonable to find the wavelengths of the two resonance modes independent of the structure angle. We further investigate the refractive index sensitivity of the dimer structures with various structure angles. The refractive index of the surrounding medium was set as $n=1.28,1.30,1.32$ in the index sensitivity calculations. With it evaluated and plotted as a function of $\theta$ (Fig. 5d), we find the sensitivity is independent of the structure angle.

\section{Conclusions}

In conclusion, we performed a systematic study on the angleresolved plasmonic properties of single gold nanorod dimer structures both experimentally and theoretically. Strong plasmonic coupling occurs in these dimer structures, which results in the hybridized bonding and antibonding resonance modes. Single-particle dark-field scattering experiments indicate that the modes both are highly sensitive to the angle between the excitation polarization and the orientation of the dimer. The scattering intensity follows a typical $\cos ^{2} \varphi$ dependence due to the decomposition of the excitation electric field vector along either the bisector of the dimer for the radiative antibonding mode or its orthogonal direction for the radiative bonding mode. Effect of the angle between the two nanorods in the dimer on the scattering is also investigated by combining SEM and single-particle scattering spectroscopy. Both analytical and numerical methods are used in the calculations. The resonance wavelengths as well as the refractive index sensitivities are found independent of the structure angle. The calculated results are in good agreement with the measurements. Knowledge gained is helpful for the design of more complicated plasmonic structures, such as Fano and chiral structures. It is of particular interest for applications because these dimer structures can be used as building blocks in plasmon-based optical and optoelectronic devices.

Acknowledgments This work is supported by the National Natural Science Foundation of China (NSFC) (grant no. 61178014, 11274231, and 21271181), the key project of the Ministry of Education of China under grant 109061, Ministry of Science and Technology of China (Inter-governmental S\&T Cooperation Project, grant no. 6-10), and Thousand Youth Talents Program of China.

Open Access This article is distributed under the terms of the Creative Commons Attribution License which permits any use, distribution, and reproduction in any medium, provided the original author(s) and the source are credited.

\section{References}

1. S. Link, M.A. El-Sayed, Optical properties and ultrafast dynamics of metallic nanocrystals. Annu. Rev. Phys. 54(1), 331-366 (2003). doi:10.1146/annurev.physchem.54.011002.103759
2. S. Biswas, J. Duan, D. Nepal, R. Pachter, R. Vaia, Plasmonic resonances in self-assembled reduced symmetry gold nanorod structures. Nano Lett. 13(5), 2220-2225 (2013). doi:10.1021/n14007358

3. B. Luk'yanchuk, N.I. Zheludev, S.A. Maier, N.J. Halas, P. Nordlander, H. Giessen, C.T. Chong, The fano resonance in plasmonic nanostructures and metamaterials. Nat. Mater. 9(9), 707-715 (2010). doi:10.1038/nmat2810

4. L. Shao, K.C. Woo, H. Chen, Z. Jin, J. Wang, H.Q. Lin, Angleand energy-resolved plasmon coupling in gold nanorod dimers. ACS Nano 4(6), 3053-3062 (2010). doi:10.1021/nn100180d

5. R. Iovine, L.L. Spada, L. Vegni, Optical properties of modified nanorod particles for biomedical sensing. Magn. IEEE Trans. 50(2), 169-172 (2014). doi:10.1109/TMAG.2013.2284552

6. H. Chen, L. Shao, Q. Li, J. Wang, Gold nanorods and their plasmonic properties. Chem. Soc. Rev. 42(7), 2679-2724 (2013). doi:10.1039/C2CS35367A

7. G. Baffou, R. Quidant, Nanoplasmonics for chemistry. Chem. Soc. Rev. 43(11), 3898-3907 (2014). doi:10.1039/C3CS60364D

8. D. Gomez, T. Davis, A. Funston, Plasmonics by design: design principles to structure-function relationships with assemblies of metal nanoparticles. J. Mater. Chem. C 2(17), 3077-3087 (2014). doi:10.1039/C3TC32041C

9. J.N. Li, T.Z. Liu, H.R. Zheng, F. Gao, J. Dong, Z.L. Zhang, Z.Y. Zhang, Plasmon resonances and strong electric field enhancements in side-by-side tangent nanospheroid homodimers. Opt. Express 21(14), 17176-17185 (2013). doi:10.1364/OE.21.017176

10. R. Fernández-García, Y. Sonnefraud, A.I. Fernández-Domínguez, V. Giannini, S.A. Maier, Design considerations for near-field enhancement in optical antennas. Contemp. Phys. 55(1), 1-11 (2014). doi:10.1080/00107514.2013.850788

11. P.K. Jain, S. Eustis, M.A. El-Sayed, Plasmon coupling in nanorod assemblies: optical absorption, discrete dipole approximation simulation, and exciton-coupling model. J. Phys. Chem. B 110(37), 18243-18253 (2006). doi:10.1021/jp063879z

12. J. Kumar, X. Wei, S. Barrow, A.M. Funston, K.G. Thomas, P. Mulvaney, Surface plasmon coupling in end-to-end linked gold nanorod dimers and trimers. Phys. Chem. Chem. Phys. 15(12), 4258-4264 (2013). doi:10.1039/C3CP44657C

13. S. Biswas, J. Duan, D. Nepal, K. Park, R. Pachter, R.A. Vaia, Plasmon induced transparency in the visible via self-assembled gold nanorod heterodimers. Nano Lett. 13(12), 6287-6291 (2013). doi:10.1021/n1403911z

14. L.V. Brown, H. Sobhani, J.B. Lassiter, P. Nordlander, N.J. Halas, Heterodimers: plasmonic properties of mismatched nanoparticle pairs. ACS Nano 4(2), 819-832 (2010). doi:10.1021/nn9017312

15. C. Tabor, D. Van Haute, M.A. El-Sayed, Effect of orientation on plasmonic coupling between gold nanorods. ACS Nano 3(11), 3670-3678 (2009). doi:10.1021/nn900779f

16. M.W. Chu, V. Myroshnychenko, C.H. Chen, J.P. Deng, C.Y. Mou, F.J. García de Abajo, Probing bright and dark surfaceplasmon modes in individual and coupled noble metal nanoparticles using an electron beam. Nano Lett. 9(1), 399-404 (2009). doi: $10.1021 / \mathrm{n} 1803270 x$

17. M.S.M. Saifullah, T. Ondarçuhu, D.K. Koltsov, C. Joachim, M.E. Welland, A reliable scheme for fabricating sub-5 nm co-planar junctions for single-molecule electronics. Nanotechnology 13(5), 659 (2002). doi:10.1088/0957-4484/13/5/323

18. L.S. Slaughter, Y. Wu, B.A. Willingham, P. Nordlander, S. Link, Effects of symmetry breaking and conductive contact on the plasmon coupling in gold nanorod dimers. ACS Nano 4(8), 4657-4666 (2010). doi:10.1021/nn1011144

19. S. Panaro, A. Nazir, C. Liberale, G. Das, H. Wang, F. De Angelis, R.P. Zaccaria, E. Di Fabrizio, A. Toma, Dark to bright mode conversion on dipolar nanoantennas: a symmetry-breaking approach. ACS Photonics 1(4), 310-314 (2014). doi:10.1021/ ph500044w 
20. W.S. Chang, J.W. Ha, L.S. Slaughter, S. Link, Plasmonic nanorod absorbers as orientation sensors. P. Natl. Acad. Sci. USA 107(7), 2781-2786 (2010). doi:10.1073/pnas.0910127107

21. C. Sönnichsen, A.P. Alivisatos, Gold nanorods as novel nonbleaching plasmon-based orientation sensors for polarized singleparticle microscopy. Nano Lett. 5(2), 301-304 (2004). doi:10. 1021/n1048089k

22. W.H. Ni, H.J. Chen, J. Su, Z.H. Sun, J.F. Wang, H.K. Wu, Effects of dyes, gold nanocrystals, $\mathrm{pH}$, and metal ions on plasmonic and molecular resonance coupling. J. Am. Chem. Soc. 132(13), 4806-4814 (2010). doi:10.1021/ja910239b

23. W.H. Ni, R.A. Mosquera, J. Perez-Juste, L.M. Liz-Marzan, Evidence for hydrogen-bonding-directed assembly of gold nanorods in aqueous solution. J. Phy. Chem. Lett. 1(8), 1181-1185 (2010). doi:10.1021/jz1002154

24. P. Pramod, K.G. Thomas, Plasmon coupling in dimers of $\mathrm{Au}$ nanorods. Adv. Mater. 20(22), 4300-4305 (2008). doi:10.1002/ adma.200703057

25. J. Kern, S. Grossmann, N.V. Tarakina, T. Hackel, M. Emmerling, M. Kamp, J.S. Huang, P. Biagioni, J.C. Prangsma, B. Hecht, Atomic-scale confinement of resonant optical fields. Nano Lett. 12(11), 5504-5509 (2012). doi:10.1021/nl302315g

26. W. Ni, X. Kou, Z. Yang, J. Wang, Tailoring longitudinal surface plasmon wavelengths, scattering and absorption cross sections of gold nanorods. ACS Nano 2(4), 677-686 (2008). doi:10.1021/ nn7003603

27. Y. Wu, P. Nordlander, Finite-difference time-domain modeling of the optical properties of nanoparticles near dielectric substrates. J. Phy. Chem. C 114(16), $7302-7307$ (2010). doi:10. 1021/jp908980d

28. P. Yang, K.N. Liou, Finite-difference time domain method for light scattering by small ice crystals in three-dimensional space. J. Opt. Soc. Am. A 13(10), 2072-2085 (1996). doi:10.1364/ JOSAA.13.002072

29. E. Prodan, C. Radloff, N.J. Halas, P. Nordlander, A hybridization model for the plasmon response of complex nanostructures. Science 302(5644), 419-422 (2003). doi:10.1126/science. 1089171

30. H.C. van de Hulst, Light scattering by small particles (Dover Publications, New York, 1957)

31. R. Gans, The state of ultramicroscopic silver particles. Ann. Phys-Berlin 47(10), 270-284 (1915). doi:10.1002/andp. 19153521006

32. P.B. Johnson, R.W. Christy, Optical constants of noble metals. Phy. Rev. B 6(12), 4370-4379 (1972). doi:10.1103/PhysRevB.6. 4370

33. B.T. Draine, The discrete-dipole approximation and its application to interstellar graphite grains. Astro Phys. J. 333(2), 848-872 (1988). doi:10.1086/166795

34. K. Liu, A. Ahmed, S. Chung, K. Sugikawa, G. Wu, Z. Nie, R. Gordon, E. Kumacheva, In situ plasmonic counter for polymerization of chains of gold nanorods in solution. ACS Nano 7(7), 5901-5910 (2013). doi:10.1021/nn402363p

35. Z. Sun, W. Ni, Z. Yang, X. Kou, L. Li, J. Wang, pH-controlled reversible assembly and disassembly of gold nanorods. Small 4(9), 1287-1292 (2008). doi:10.1002/smll.200800099 